\title{
Left ventricular performance and related haemodynamic changes in Prinzmetal's variant angina pectoris
}

\author{
M. Guazzi, A. Polese, C. Fiorentini, F. Magrini, and C. Bartorelli \\ From the Istituto di Clinica Medica II and Istituto di Ricerche Cardiovascolari, \\ University of Milan, Milan, Italy
}

Attacks of Prinzmetal's variant form of angina pectoris are spontaneous, recur cyclically, and present unequivocal electrocardiographic alterations: they are ideal for a detailed haemodynamic study.

Four patients with this form of angina were investigated. In each of them episodes occurred of electrocardiographic abnormalities either accompanied or unaccompanied by pain. During the same session, the cardiogram, heart rate, arterial pressure, and right atrial pressure were continuously recorded during periods ranging from 5 to 7 hours in each patient. Cardiac output was measured at selected times. Left ventricular ejection time, isovolumic contraction time, mean rate of isovolumic pressure development, and mean systolic ejection rate were also determined.

In the 38 recorded anginal episodes, no circulatory change preceded the cardiographic modifications. From the onset of the electrocardiographic abnormalities to the beginning of their reversion, the following circulatory events were observed: (I) obvious reduction of cardiac output; (2) arterial hypotension; (3) lengthening of isovolumic contraction time and mean rate of isovolumic pressure development; (5) reduction of mean systolic ejection rate. It is concluded: (I) that no circulatory factor interfering with work or oxygen consumption of the heart is responsible for eliciting these anginal episodes; (2) that conspicuous left ventricular impairment occurs during the increasing and steady period of the electrocardiographic abnormalities.

As the electrocardiogram started reverting to the pre-attack aspect, cardiac performance rapidly improved and, after a 'supernormal' phase, returned in about 2 minutes to basal levels. It is possible that this phase is dependent on a temporary sympathetic compensatory mechanism.

No significant qualitative differences were detected between the circulatory pattern of various anginal episodes. The difference was mainly quantitative and the magnitude of the haemodynamic changes correlated well with the degree of the electrocardiographic abnormalities. Pain, when present, seemed just a concomitant symptom not significantly interfering with the circulatory changes associated with the episodes of this form of angina pectoris.

The circulatory changes associated with exertion or drug-induced angina pectoris have been the subject of several reports. The available haemodynamic data concerning spontaneous angina pectoris are more often the result of occasional observations than of a systematic study. The latter has been beset by technical problems: the rapidity of the circulatory changes associated with the onset, presence, and disappearance of the anginal attacks necessitates continuous recording of the electrocardiogram and of the circulatory parameters. In addition, finding patients with Received 6 April 1970. spontaneous anginal episodes occurring frequently enough to provide reliable and reproducible information, is an unusual event.

It is generally agreed that major changes in the greater and lesser circulation, consistent with impaired left ventricular performance, may be related to spontaneous anginal episodes (Roughgarden and Newman, 1966; Roughgarden, 1966; Müller and Rørvik, 1958; Ross et al., 1962; Cohen et al., 1965; Gorlin, 1965; Rosland, 1969). Some important points, however, remain unsettled. Detailed information about the sequence of the circulatory events preceding the onset of 
anginal attacks as well as a correlation between the time of onset of systemic hypertension, increase in left ventricular enddiastolic pressure, and electrocardiographic abnormalities, have not been provided. It is also not clear whether anginal pain is responsible for eliciting arterial hypertension and whether the latter contributes to left ventricular impairment.

The attacks of a variant form of angina pectoris, as described by Prinzmetal et al. (1959), are spontaneous and often occur at approximately the same time each day; the related electrocardiographic changes (ST segment elevation in those leads that reflect the ischaemic area) are so well defined to make unequivocal both the beginning and the end $:$ of the anginal episodes. They are ideal for a systematic and detailed haemodynamic study.

The observations on 4 patients with this form of angina are here reported.

\section{Methods}

7 Four subjects were investigated. They each gave a history of spontaneous substernal pains of variable occurrence, during periods ranging from 3 months to 6 years. The anginal attacks had be. come more and more frequent in the days before admission. The electrocardiogram was normal in one patient (G.N.) and showed an old myocardial infarction and left ventricular hypertrophy in , another (L.M.). In the last 2 subjects (G.V. and F.T.) the electrocardiogram revealed recent infarction of the diaphragmatic and antero-septal myocardial areas, respectively. In both patients infarct occurred 6 days before admission.

Daily charting during a one-week period of the time and circumstances of the attacks indicated the hours of the day when spontaneous angina occurred most often for each individual patient. The circulatory study was carried out during those hours.

In order to prevent the possible influence of emotional factors, care was taken to make the patients familiar with the investigators as well as with the laboratory. They came to the laboratory on three consecutive days. On the first day they merely lay comfortably in bed for a few hours. On the second day they lay in bed for several hours while continuous electrocardiographic recording was made. On the third day the circulatory study was carried out; this session was of the same luration as the second day, and lasted 7 hours for zatient G.N., 6 hours for patient L.M., and 5 iours for patients G.V. and F.T.

The electrocardiographic abnormalities during vhich anginal episodes occurred (Guazzi et al., 970), can be summarized as follows: obvious ST egment elevation in the inferior leads in the first gree subjects, and in leads $V_{2}-5$ in patient F.T.

Studies were done in the fasting state, and no remedication was given. For the measurement f right atrial pressure a thin radiopaque catheter was inserted into an antecubital vein and advanced into the right atrium under fluoroscopic control. An 18-gauge Teflon catheter needle, inserted into the brachial artery, was used to sample indocyanine green for cardiac output and to monitor arterial pressure. Pressures were determined with Statham P23 De and P23 Db strain gauge transducers. Dye dilution analyses of cardiac output were performed after injection of indocyanine green $(5 \mathrm{mg}$.) into the right atrium and withdrawal from the brachial artery. Because of the numerous cardiac output measurements, the withdrawn blood was reinfused except for that small amount which was necessary for calibrating the Gilford cuvette densitometer at the end of the procedure. This was accomplished by making up three dilutions of dye in the patient's own blood. The output signal of a cuvette densitometer (Gilford) was coupled to the input of a digital computer (Gilford). The area under each dye curve was measured by a planimeter as well as a digital computer, and cardiac output was calculated by the standard Hamilton method. Systemic peripheral resistance was calculated from the formula :

SPR $\left(\right.$ dynes.sec.cm. $\left.{ }^{-5}\right)=$

$$
\frac{\text { MAP }- \text { MRAP } \times 1332 \times 60}{\mathrm{CO}(\mathrm{ml} . / \mathrm{min} .)}
$$

where MAP and MRAP are, respectively, the mean arterial and mean right atrial pressures in millimetres Hg. The electrocardiographic lead recorded was that which showed the most evident changes of $T$ wave and ST tract. Beat-to-beat variations of the heart rate were detected by a Cardio-Tach Preamplifier (Hewlett-Packard 350$3400 \mathrm{~A})$. According to the method indicated by Weissler, Harris, and Schoenfeld (I968), the duration of the left ventricular ejection time and of the isovolumic contraction time was measured from simultaneous recordings of the electrocardiogram, the phonocardiogram and, when the beginning of the upstroke and the trough of the incisura were questionable in the brachial artery tracing, the carotid arterial tracing. All the parameters, except for cardiac output, were continuously recorded on an 8-channel ink recorder (Hewlett-Packard 7868/A). The electrocardiogram was also monitored on an oscilloscope. During slow-speed paper recording the electrocardiographic pattern could be monitored by inspecting the oscilloscope tracing, which was set at a sweep speed of $25 \mathrm{~mm}$./sec. However, the anginal changes were so clear in each patient (see Fig. 2, 3, 5, and 7) as to make unequivocal the identification of the attacks of angina even at records of $\mathrm{I} \mathrm{mm}$./sec. For the measurements of the left ventricular systolic time intervals, records were obtained at a paper speed of $100 \mathrm{~mm}$./ $/ \mathrm{sec}$. A microphone was placed over the praecordium in a position optimal for recording the initial high frequency vibrations of the first and second heart sounds. Two microphones were sometimes necessary to define the initial vibrations of both sounds. The carotid pulse was recorded with a Sanborn APT/16/1 transducer. Left ventricular ejection time, from the beginning upstroke to the 
trough of the incisura of the carotid pulse tracing, and the interval between the first and second heart sound $\left(S_{1} S_{2}\right)$, from the beginning of the first sound to the onset of the aortic component of the second sound, were measured directly. The isovolumic contraction time was calculated by subtracting the left ventricular ejection time from $S_{1} S_{2}$. All intervals were calculated from the mean of measurements on 5 consecutive beats, each read to the nearest $5 \mathrm{msec}$. The mean rate of left ventricular isovolumic pressure development was determined by measuring the height of the brachial diastolic pressure and dividing it by the isovolumic contraction time. The mean systolic ejection rate was determined by dividing the stroke index by the left ventricular ejection time.

The patients held a push button for the remote control of a marker of the recording system. They

FIG. I Schematic representation of the pattern of some circulatory parameters during anginal attacks of the patient G.N. The measurements have been made at selected times indicated at top, as described under Methods. From top: AP, arterial pressure; RAP, right atrial pressure;

$H R$, heart rate; IVCT, isovolumic contraction time; MRIPD, mean rate of isovolumic pressure development; CI, cardiac index; $A T$, dye appearance time; MSEFR, mean systolic ejection rate. Together with some of the values of $C I$ the contemporary values of systemic peripheral resistence (dynes.sec.cm ${ }^{-5}$ ) are reported. The dotted area indicates the duration, the extent, the waxing, and the waning periods of ST tract elevation. See text for further details.
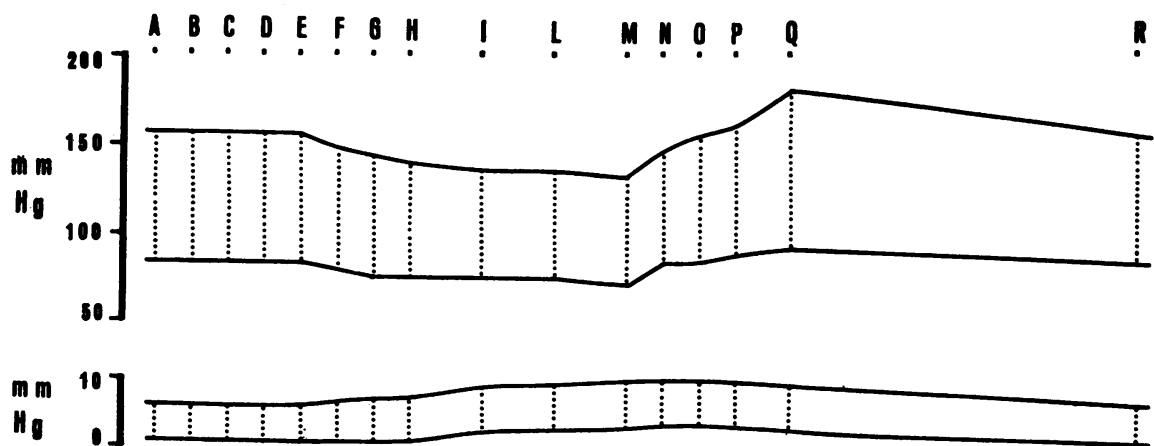

\section{b/min." $\left.{ }_{10}^{10}\right]$}
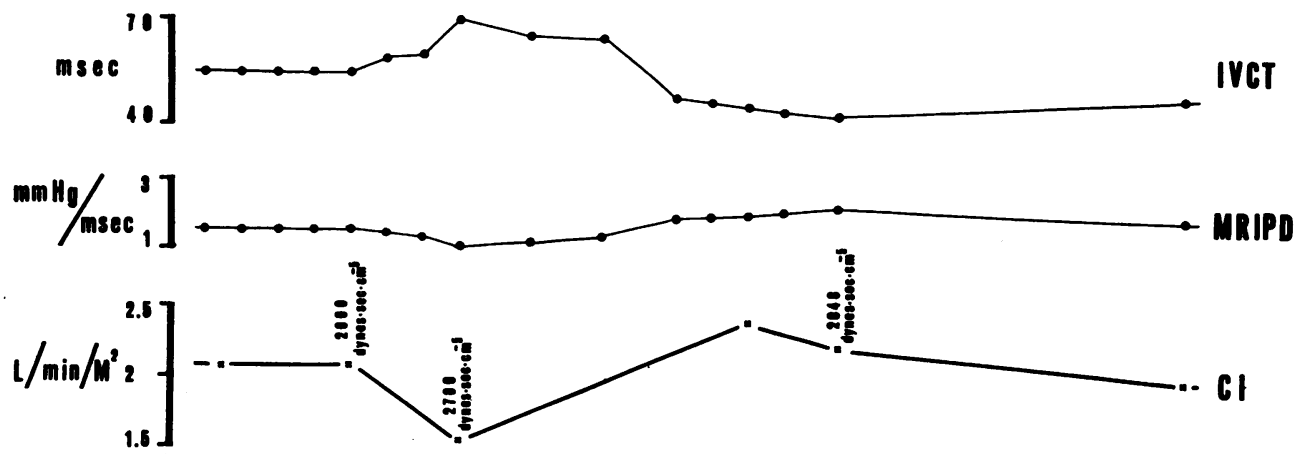

$$
\text { t11" t11" t14.8" }
$$

110.8" f11"

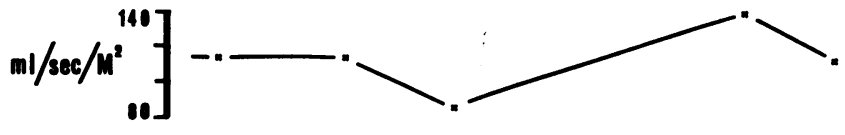

$m m+5$

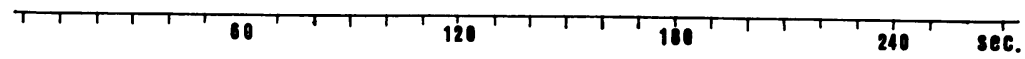


were instructed to keep it pushed for the entire duration of the pain, so that a good time relation between pain and electrocardiographic and circulatory alterations could be recorded. Each episode of electrocardiographic abnormality was followed by detailed questioning of the patient in order to assess his pain.

In each individual patient no significant difference either in the frequency or in the distribution in the anginal episodes, whether accompanied by pain or not, was detected between the circulatory and the electrocardiographic study carried out the day before (Guazzi et al., 1970). Both recording sessions were continued for the same number of hours.
From the continuous record, measurements of arterial pressure, right atrial pressure, and heart rate were made during the four Io-second intervals (indicated as A, B, C, D) which just preceded the onset of the electrocardiographic abnormalities. During the ST tract elevation measurements were taken at the following times: onset of electrocardiographic abnormality (E); waxing period $(F, G)$; beginning of the steady period $(H)$; during the steady state ( $I, L)$; beginning of the waning period $(M)$; during the waning period $(\mathrm{N}, \mathrm{O})$; at the moment when the electrocardiogram reverted to its usual appearance $(P)$; and I5 seconds (Q) and 90 seconds (R) after the electrocardiogram returned to its original appear-

FIG. 2 Patient G.N. Original record showing the waxing period and part of the steady period of an anginal episode. From top: time marker; HR, heart rate; $H S$, heart sounds; $C P$, carotid pulse tracing; II, lead II of the electrocardiogram; AP, brachial arterial pressure; $R A P$, right atrial pressure. The heavy line at the bottom indicates pain. The onset of the anginal attack is signalled by the arrow. Note the progressive elevation of the ST tract and the pattern of the $T$ wave which in the steady period appears as tall as the $R$ wave.

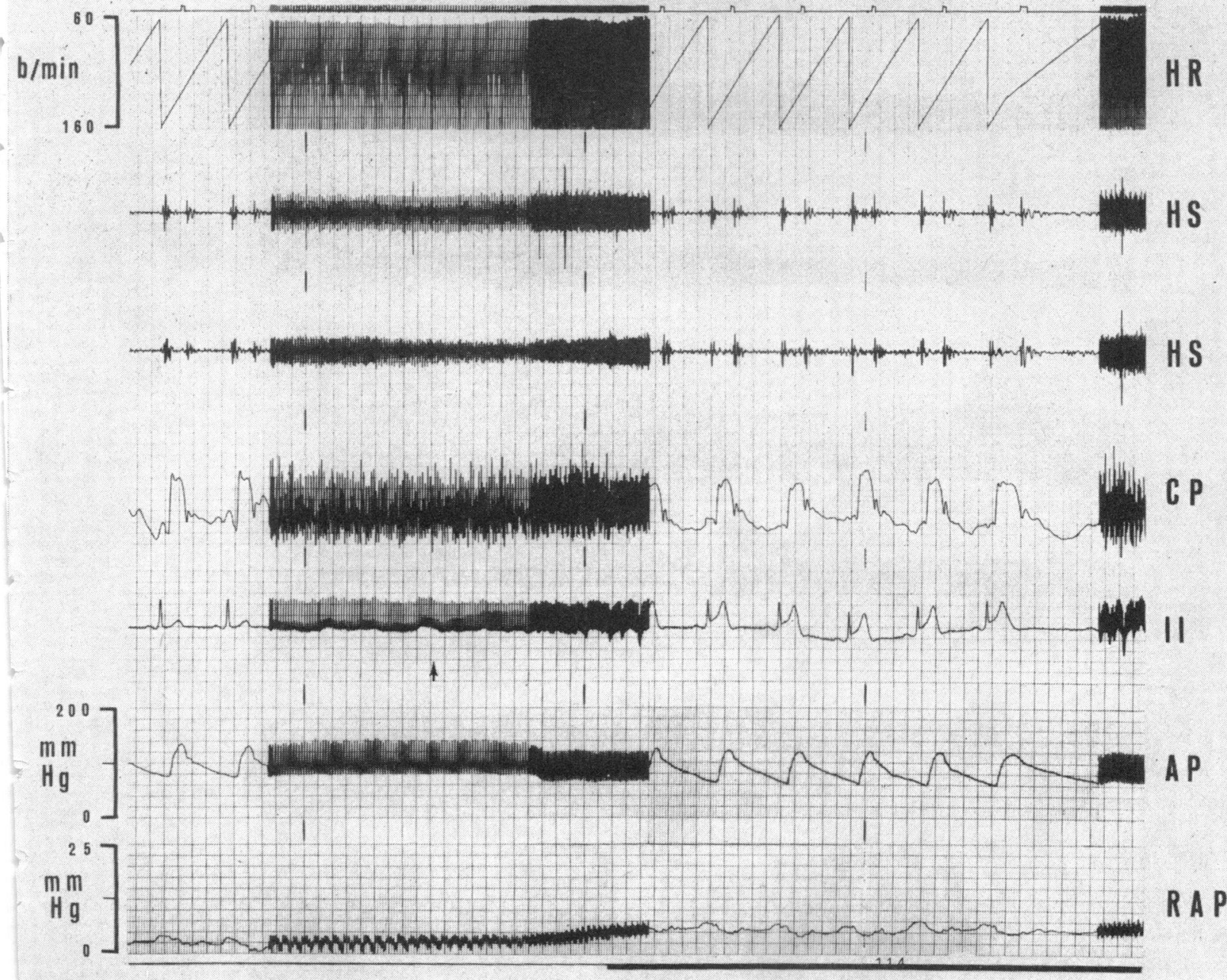


ance. In patients G.N., L.M., and F.T., records of two anginal episodes were entirely done at a paper speed of $100 \mathrm{~mm}$./ $\mathrm{sec}$. Measurements of the left ventricular ejection time and of the isovolumic contraction time were made at all the times previously indicated. Only three anginal episodes occurred during the study of the patient G.V.; none of them was recorded at high paper speed. Cardiac output was determined at the times indicated in the Figures.

\section{Results}

In the patients G.N., L.M., and F.T., the episodes of electrocardiographic alteration accompanied by pain were almost as frequent as those without pain. Because no qualitative difference, as concerns the measured parameters, was detected between the former and the latter, the related circulatory data have been pooled in a single group.

FIG. 3 Patient G.N. Original record of an anginal episode unaccompanied by pain.

Though the paper runs at a speed of $1 \mathrm{~mm}$./sec., the electrocardiographic abnormalities appear unequivocal. The onset and the end of the attack are signalled by the arrows. Abbreviations as in Fig. 2.

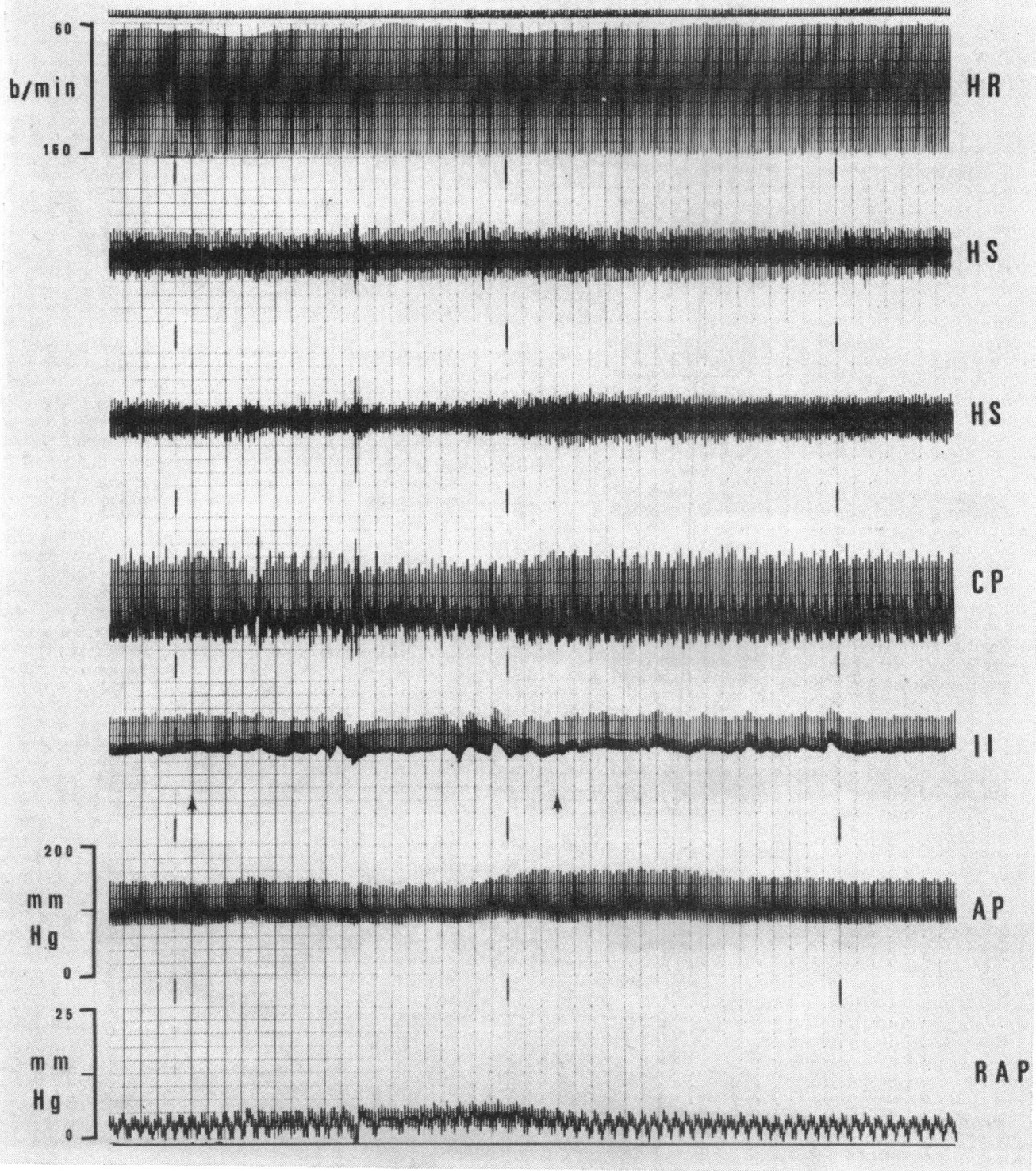


The observations made in patient G.N. have been summarized in Fig. I, showing right atrial pressure and heart rate, the means of 13 episodes of electrocardiographic abnormality analysed at the selected times described under Methods. The graph of the isovolumic contraction time represents the mean of measurements done on two high-speed records. The values of cardiac index were obtained by a single measurement of cardiac output at the times $A, E, Q, R$ and by the mean of two measurements at the times $\mathrm{H}$ and 0 .
From the onset of the abnormalities on the electrocardiogram, during the waxing and the steady period (from time $\mathrm{E}$ to time $\mathrm{M}$ ), a progressive reduction in arterial pressure was observed, and this was paralleled, at least in its initial part (no measurement was done during the late phase), by a conspicuous reduction in cardiac output (cardiac index decreased from 2100 to $1550 \mathrm{ml}$. $/ \mathrm{min}$.). The systemic peripheral resistance rose from 2000 at time $\mathrm{E}$ to 2700 dynes sec. $\mathrm{cm} .^{-5}$ at time $\mathrm{H}$. The following changes were simultaneously observed: (I) increase in right atrial pressure;

FIG. 4 Patient L.M. Schematic representation of the pattern of some circulatory parameters during anginal episodes. See text for details. Abbreviations as in Fig. I.
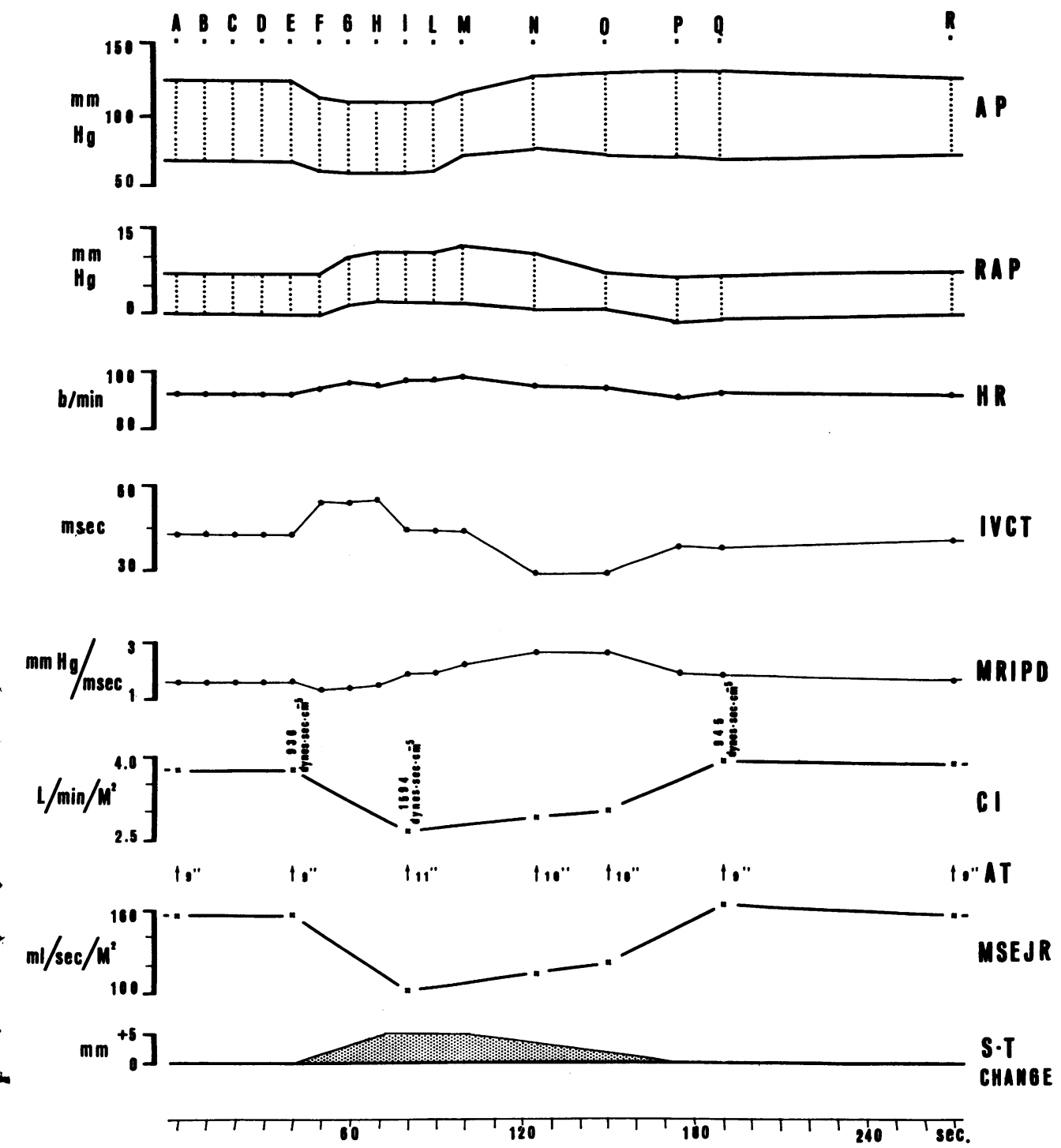
(2) prolongation of left ventricular isovolumic contraction time; (3) reduction in mean rate of isovolumic pressure development; (4) decrease in mean systolic ejection rate; and (5) lengthening in dye appearance time.

At the beginning of the waning period, arterial pressure progressively increased; it reached, a few seconds after the complete reversion of the electrocardiographic abnormality, diastolic and systolic levels much higher than those present before the onset of the angina. Cardiac output showed a parallel pattern. The systemic peripheral resistance returned to the level before the attack. At the same time as the electrocardiographic reversion, right atrial pressure started to decrease, progressively returning to normal values. The following changes were also detected during this period of electrocardiographic reversion: the isovolumic contraction time and the dye appearance time became shorter, as compared to the pre-attack periods, and the mean

FIG. 5 Patient L.M. Original record of an episode of angina pectoris. The heavy line at the bottom indicates pain. The arrows signal the onset and the end of the electrocardiographic abnormalities. Abbreviations as in Fig. 2.

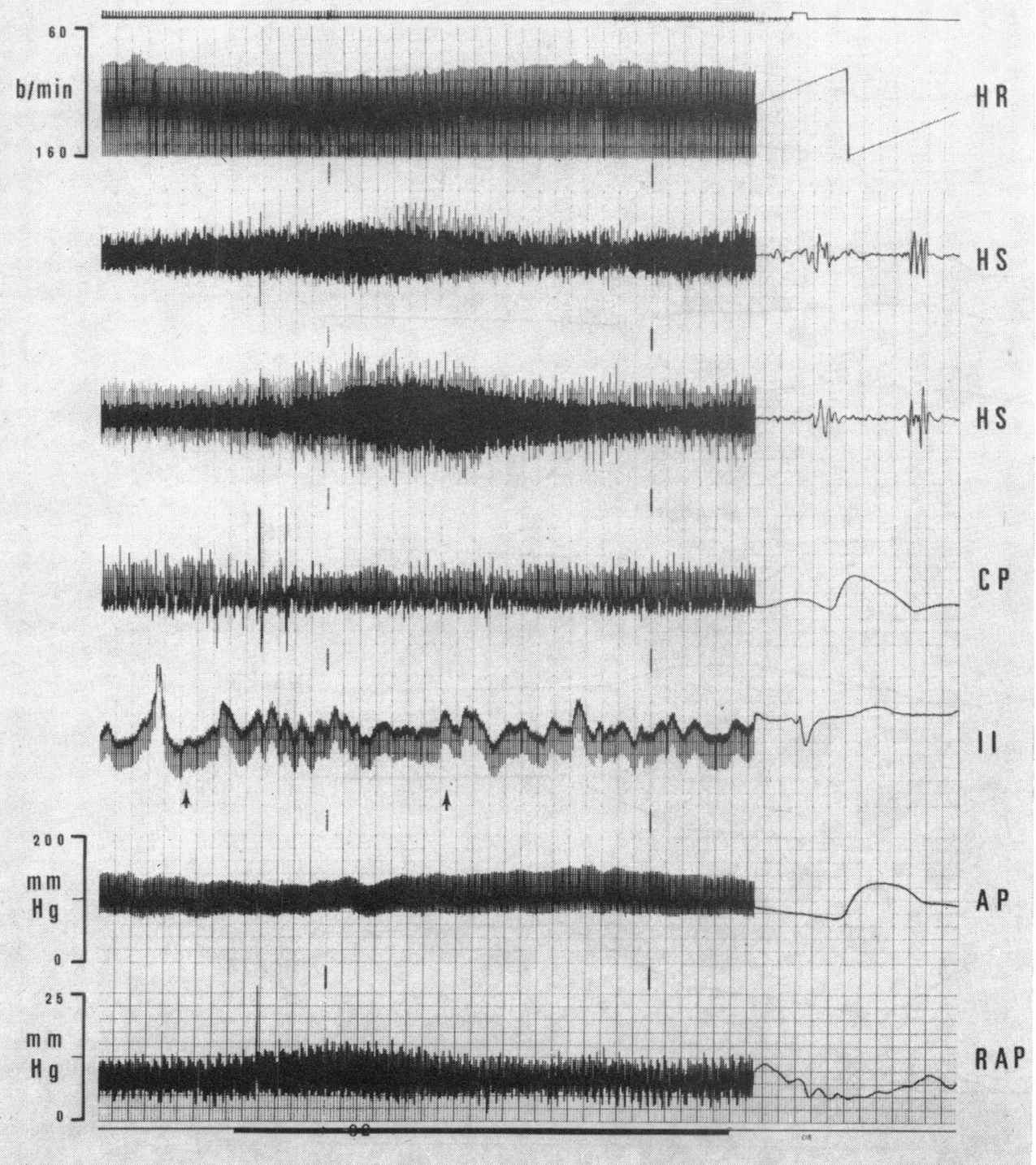


systolic ejection rate and mean rate of isovolumic pressure development rose over control values. After this 'supernormal' phase, all the parameters returned to basal levels (time $R$ ) within about 2 minutes of the complete disappearance of the electrocardiographic abnormalities.

Fig. 2 and 3 reproduce original records from this same patient. In Fig. 2 the paper speed, initially $25 \mathrm{~mm}$./sec., has been switched to lower speeds, and in order to make clearer changes in the electrocardiogram, pressures, and heart sounds, it was switched back to $25 \mathrm{~mm}$./sec. The records of the electrocardiogram, carotid pulse, and heart sounds give an idea of how easily the measurement of the left ventricular systolic time intervals could be done. Fig. 2 and 3 show the electrocardiographic and circulatory differences between episodes accompanied by pain (Fig. 2) and those without pain (Fig. 3). The former is longer lasting with more conspicuous electrocardiographic abnormalities (the $T$ wave becoming as tall as the $R$ wave) and larger atrial and arterial pressure changes. In the latter, which lasts just I minute and 30 seconds, the $T$ wave is $3 \mathrm{~mm}$. shorter than the $R$ wave and the pressure changes are smaller.

Fig. 4, summarizing the circulatory pattern

FIG. 6 Patient F.T. Schematic representation of the pattern of some circulatory parameters during anginal episodes. See text for details. Abbreviations as in Fig. I.
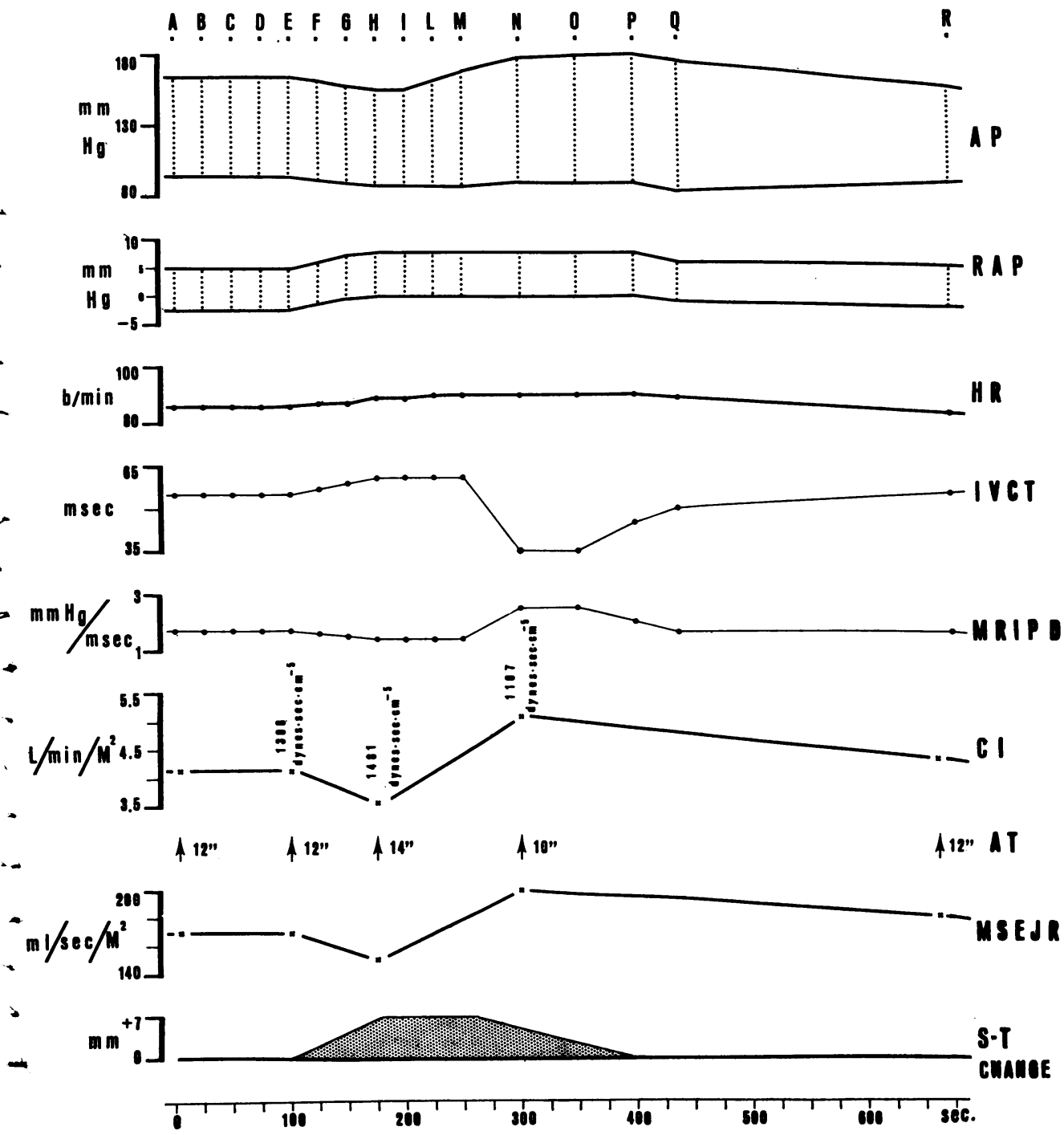
of patient L.M., presents graphs of arterial pressure and heart rate representing the mean of 15 attacks analysed at selected times. The data concerning the isovolumic contraction time and cardiac index were obtained as described for the previous patient. A comparison of this Fig. 4 with Fig. I shows that left ventricular performance and the related haemodynamic changes behave, in the two patients, in a very similar fashion. The only qualitative difference is in the heart rate. During anginal attacks, in fact, pulse rate slightly decreased in G.N. and moderately increased in L.M. The similarities in haemodynamic changes is also shown in Fig. 5, which reproduces an original record taken during an episode of angina of patient L.M. In this patient no remarkable difference, both in the electrocardiogram and circulatory pattern, was detected between the episodes with pain and those without pain.

Patient F.T. had a recent antero-septal myocardial infarction. When he was free of pain, his electrocardiogram showed $Q$ waves in leads VI-2, slight ST segment elevation in leads VI-4, and deep $T$ wave inversion in leads VI-5. During 5 hours of continuous recording, electrocardiographic changes occurred in 7 instances: only 3 of them were accompanied by aching substernal pain, whereas, on 4 occasions, electrocardiographic abnormalities were recorded without the patient suffering any pain. The electrocardiographic and circulatory abnormalities in the absence of pain were shorter in duration and a little less obvious, though still very clear, as compared to the changes recorded during painful attacks. In Fig. 7, the waning phase

FIG. 7 Patient F.T. Original record showing the waning period of an anginal attack accompanied by pain as shown by the heavy line on the bottom. From the top: time marker; $H R$, heart rate; dye dilution curve for determination of cardiac output; $H S$, heart sounds; leads $V_{4}$ and $V 5 ; A P$, arterial pressure; $R A P$, right atrial pressure. The end of the episode is signalled by the arrowheads. Note the progressive reduction of the ST segment elevation and the changes in the $T$ wave which gradually becomes negative and reverts to its usual appearance (the patient has recent antero-septal myocardial infarction).

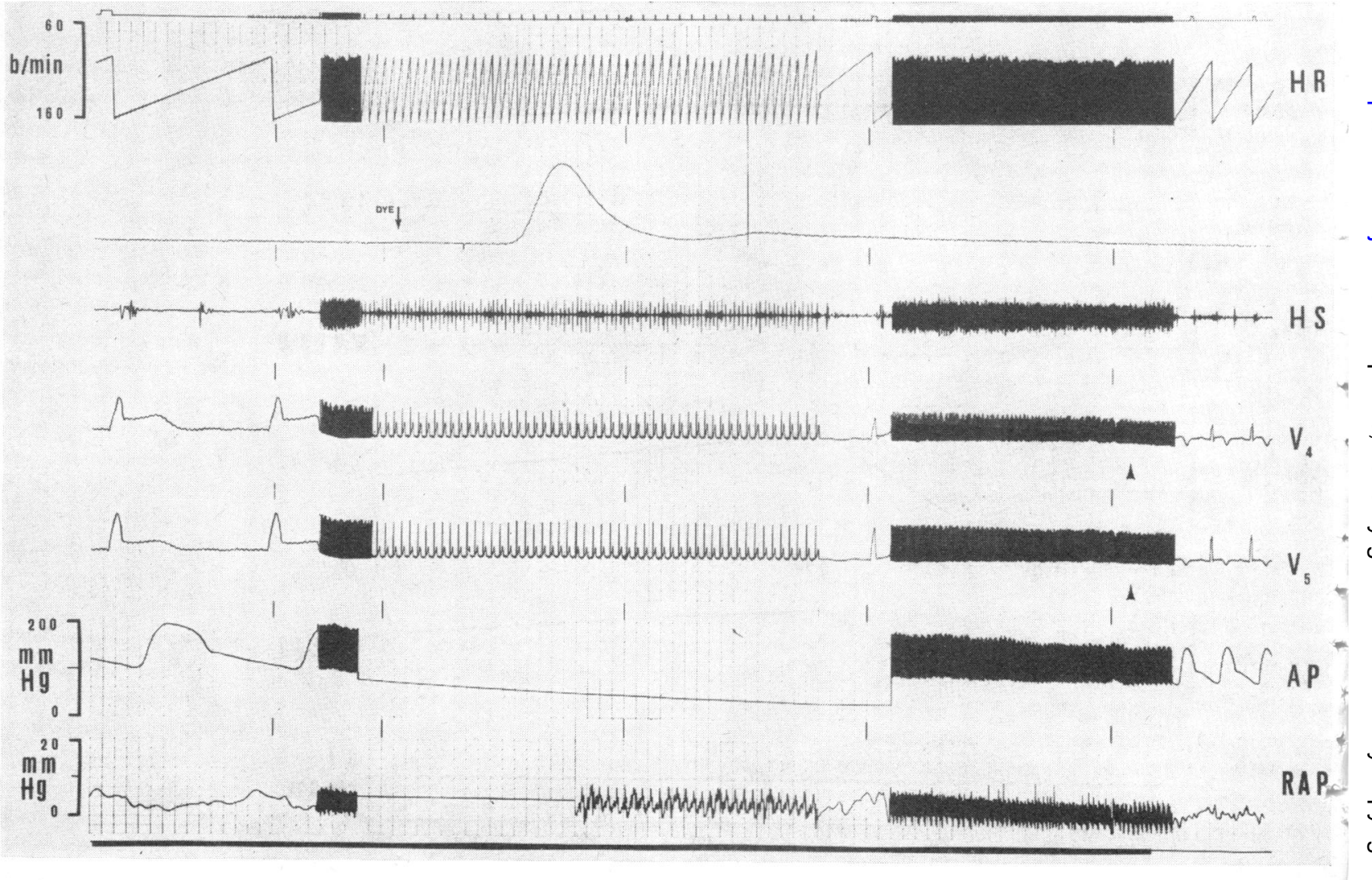


of one of the anginal attacks of this patient is reproduced: leads $\mathrm{V}_{4}$ and $\mathrm{V}_{5}$ are recorded. ST segment elevation progressively reduced and the upright $T$ wave became deeply inverted and returned to its appearance before the attack. In this patient during angina the measured circulatory parameters behaved, as shown in Fig. 6 and 7, in a manner similar to the previous two patients. Graphic representations are shown in Fig. 6. A single determination of cardiac output was performed at times $\mathrm{A}, \mathrm{E}, \mathrm{H}, \mathrm{N}$, and $\mathrm{R}$.

The circulatory study of the second patient (G.V.) with recent myocardial infarction, is incomplete because of the small number of anginal episodes which occurred during the study. None of these was recorded at high paper speed, and therefore information about left ventricular ejection time, isovolumic contraction time, mean systolic ejection rate, and mean rate of isovolumic pressure development is lacking. However, because arterial pressure, right atrial pressure, cardiac output (measured at times A, I, and P), and dye appearance time each showed the same pattern as in the other patients, it seems likely that no qualitative differences exist between the circulatory pattern of this patient and that of the previous ones.

\section{Discussion}

As already mentioned, during the circulatory study anginal episodes showed in each individual patient a frequency very similar to that observed on the previous day, when only the electrocardiogram was recorded. This fact, together with the occurrence of a large number of episodes of electrocardiographic abnormalities not perceived by the patients, makes it unlikely that stress and emotional factors have interfered with the circulatory changes observed.

In no anginal attack did haemodynamic changes precede the electrocardiographic alterations: the former always began at the same time as the latter. It is most unlikely, therefore, that this form of angina is secondary to circulatory modifications which acutely

- increase work or oxygen consumption of the heart.

- As regards the circulatory pattern, between the 38 recorded anginal episodes no major qualitative differences were detected. Differences were mainly quantitative, the magnitude of the haemodynamic changes correlating well

- with the degree of abnormality on the electrocardiogram. Though in three patients

- pain reports usually accompanied longer lasting and more obvious electrocardiographic modifications, no constant time relation was detected between pain and haemodynamic events. Moreover, one patient (L.M.), who invariably showed the same magnitude of electrocardiographic modifications, also had equal circulatory changes both in the presence and in the absence of pain. Therefore, pain seems to be a concomitant symptom which does not significantly interfere with the circulatory changes related to the episodes of this form of angina.

A reduction of cardiac output of a magnitude such as we observed in these patients suggests acute heart failure. A prolongation of the isovolumic contraction time has consistently been observed in the failing left ventricle by Weissler et al. (1968). In previous reports it was demonstrated that the maximal rate of isometric pressure increase is reduced in patients with left (Gleason and Braunwald, I962) or right (Jezek, I966) ventricular failure. Braunwald, Sarnoff, and Stainsby (1958) noted in the failing heart a decrease in mean systolic ejection rate. Therefore, the modifications reported here of isovolumic contraction time, mean systolic ejection rate, and mean rate of isovolumic pressure development, in good agreement with the pattern of the cardiac output, indicate that during the periods of anginal electrocardiographic abnormalities, left ventricular performance is much impaired. Sarnoff and Berglund (1954) noted, in the dog, that the artificial reduction of coronary blood flow had a depressive influence on Starling's law of the left ventricle.

The constant increase of right atrial pressure seen in our patients is probably related to the acute failure of the heart.

The systemic peripheral resistance was higher during anginal attacks than in the control phases, and the fall of blood pressure was therefore due only to a reduction of cardiac output.

Another point deserves some comment. As soon as the electrocardiogram began to revert to its original appearance, some of the measured parameters rapidly improved and overshot their basal levels. Calculated peripheral resistance indicates that the overshoot of blood pressure is entirely attributable to an acute and striking increase in cardiac output. If the improvement in cardiac performance were exclusively due to a restored oxygen supply, one would expect reversion to normal of cardiac performance, without a 'supernormal' phase. Hypothetically, this phase of increased myocardial contractility could be related to Starling's mechanism (higher filling pressure), or to reflex sympathetic stimulation, or to both. These mechanisms could 
become effective only after myocardial oxygen supply has been restored. As regards the sympathetic stimulation, Malliani, Schwartz, and Zanchetti (1969) have recently shown in the cat, by electrophysiological means, that experimental coronary occlusion elicits a cardiac sympathetic reflex.

In the majority of the circulatory studies of spontaneous anginal episodes so far reported, detailed clinical and electrocardiographic information about the form of angina in question has been lacking. The fact that attacks of Prinzmetal's angina are spontaneous does not imply that all spontaneous anginal episodes must show a haemodynamic pattern similar to the ones here described. Therefore, extension of the circulatory information concerning this particular form of angina to all spontaneous attacks can be misleading, though some common aspect between the present data and those previously reported appears evident.

\section{References}

Braunwald, E., Sarnoff, S. J., and Stainsby, W. N. (1958). Determinants of duration and mean rate of ventricular ejection. Circulation Research, 6, 319.

Cohen, L. S., Elliott, W. C., Rolett, E. L., and Gorlin, R. (1965). Hemodynamic studies during angina pectoris. Circulation, 31, 409.

Gleason, W. L., and Braunwald, E. (1962). Studies on the first derivative of the ventricular pressure pulse in man. fournal of Clinical Investigation, 41, 80.

Gorlin, R. (1965). Pathophysiology of cardiac pain. Circulation, 32, 138.
Guazzi, M., Fiorentini, C., Polese, A., and Magrini, F. (1970). Continuous electrocardiographic recording in Prinzmetal's variant angina pectoris. A report of four cases. British Heart fournal, 32, 6I I.

Jezek, V. (I966). Rate of right ventricular isometric pressure increase in the patients with chronic cor pulmonale studied by the ventricular pressure derivative curves. Cardiologia, 48, 46I.

Malliani, A., Schwartz, P. J., and Zanchetti, A. (1969). A sympathetic reflex elicited by experimental coronary occlusion. American fournal of Physiology, 217, 703.

Müller, O., and Rørvik, K. (1958). Haemodynamic consequences of coronary heart disease; with observations during anginal pain and on the effect of nitroglycerine. British Heart fournal, 20, 302.

Prinzmetal, M., Kennamer, R., Merliss, R., Wada, T., and Bor, N. (1959). Angina pectoris. I. A variant form of angina pectoris. American fournal of Medicine, 27, 375.

Rosland, G. A. (1969). Haemodynamic observations during spontaneous angina pectoris. British Heart Fournal, 31, 523.

Ross, R. S., Schwartz, N., Gaertner, R. A., and Friesinger, G. C. (1962). Electrocardiographic and haemodynamic observations during selective coronary cineangiocardiography. Fournal of Clinical Investigation, 41, 1395.

Roughgarden, J. W. (I966). Circulatory changes associated with spontaneous angina pectoris. American fournal of Medicine, 41, 947.

$\longrightarrow$, and Newman, E. V. (1966). Circulatory changes during the pain of angina pectoris. 1772-1965-A critical review. Americal fournal of Medicine, 41, 935.

Sarnoff, S. J., and Berglund, E. (1954). Ventricular function. I. Starling's law of the heart studied by means of simultaneous right and left ventricular function curves in the dog. Circulation, 9, 706.

Weissler, M. A., Harris, W. S., and Schoenfeld, C. D. (1968). Systolic time intervals in heart failure in man. Circulation, 37, 149. 